\title{
Erfolgsfaktor Data Governance
}

\author{
Christiana Klingenberg, Kristin Weber
}

Von Master Data Management (MDM) versprechen sich Unternehmen Effizienz, Transparenz und Risikominimierung im Umgang mit ihren Stammdaten. MDM soll dazu beitragen, Stammdaten als „Asset“ im Unternehmen zu bewirtschaften. Der vorliegende Beitrag liefert praktische Tipps, wie MDM-Implementierungen nachhaltig gestaltet werden können, damit die Daten einen Beitrag zum Unternehmenserfolg leisten. Er stellt das qualitätsorientierte Data Governance Framework vor. Das Framework stellt sicher, dass bei einer Implementierung alle Aspekte von MDM adressiert werden inkl. strategischer und organisatorischer Fragestellungen. Die konsequente Ausrichtung an der Datenqualität sorgt dafür, dass alle Unternehmensbereiche

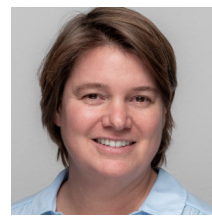

Dr. Christiana Klingenberg ist Principal Business Consultant bei msg systems. Dort berät sie Kunden zu Datenqualität und Data Governance von Stammdaten. Sie erarbeitet mit Unternehmen nachhaltige Datenmanagement-Maßnahmen und setzt diese um.

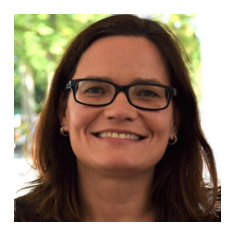

Prof. Dr. Kristin Weber ist Professorin an der HAW Würzburg-Schweinfurt (FHWS). Sie lehrt, schreibt, referiert und berät zu IT-Organisation, IT-Management, Data Governance, Datenqualität, Stammdatenmanagement, Information Security Awareness und ISMS. Stammdaten nutzenstiftend einsetzen können.

\section{Treiber MDM- Implementierung}

Es gibt verschiedene Gründe, die für die Einführung eines Stammdatenmanagement-Systems (Master Data Management, MDM) sprechen [1]. Oft ist es der Bedarf nach einer Konsolidierung der stammdatenhaltenden Systeme bzw. der darin gehaltenen Daten. Auch die Ablösung eines Systems, welches aktuelle Anforderungen nicht mehr erfüllt, kann ein Grund für die Implementierung eines neuen MDM-Systems sein. In manchen Fällen ist es auch der Datenschutz, der das datenschutzkonforme Management von personenbezogenen Daten erfordert. Oft steht der Wunsch nach mehr Effizienz beim Arbeiten mit den Daten und mehr Transparenz über die im Unternehmen vorhandenen Daten im Vordergrund. Oder es geht um die Minimierung von RisiIn diesem Beitrag lesen Sie:

- welche Bedeutung das qualitätsorientierte Data Governance Framework bei der Einführung von MDM-Systemen hat,

- welche Maßnahmen hilfreich sind bei einer MDM-Implementierung,

- welche Relevanz die Datenqualität für ein nachhaltiges Stammdatenmanagement hat. ken bei datenbasierten Entscheidungen im Bereich Business Intelligence und Predictive Analytics. Letztendlich können gute Stammdaten auch die Grundlage für Initiativen im Bereich der künstlichen Intelligenz sein.

Somit ist die Implementierung eines MDM-Systems mit einer Reihe großer Erwartungen verknüpft. Da die Anschaffung, die Implementierung und das Rollout von MDM-Systemen ressourcenhungrige Vorhaben sind, sollte eine nachhaltige und erfolgsversprechende Vorgehensweise gewählt werden.

\section{Größte Projektrisiken}

In den Medien wird immer wieder über gescheiterte MDM-Projekte berichtet [2]. Die Gründe im Einzelnen sind komplex und vielfältig. Oft sind es unklare Anforderungen an das MDMSystem bzw. dessen Implementierung. Fehlende Abstimmung zwischen den Fachbereichen, ungeklärte Zuständigkeiten oder schlicht fehlendes Commitment und fehlende Change-Management-Prozesse sind nur ein paar Gründe, die Projekte in die Länge treiben. Als Folge davon wachsen die Kosten, die Unzufriedenheit über den Projektstand steigt und im schlimmsten Fall scheitert das ganze MDM-Projekt. Nach der Implementierung fehlt die Akzep- tanz für das neue System, die Prozesse sind nicht darauf zugeschnitten und die Datenqualität ist (immer noch) nicht zufriedenstellend. Die Stimmung bei den Mitarbeitern in Bezug auf Stammdatenmanagement wird schlechter und eine grundsätzlich gute Initiative ist gescheitert.

\section{Bedeutung von Data Governance}

Die gute Nachricht ist: Das muss nicht sein. Denn wer sich von vornherein um den passenden Rahmen und entsprechende Vorgaben kümmert, kann viele Missverständnisse vermeiden und für Transparenz bei der Implementierung sorgen. Data Governance bietet einen Rahmen mit verschiedenen Handlungsebenen, der eine erfolgreiche MDM-Implementierung unterstützt und den Nutzen nach dem Go-live nachhaltig sichert. Die unterschiedlichen Aspekte von Data Governance und damit auch von Stammdatenmanagement werden in sogenannten Data Governance Frameworks dargestellt. Diese Frameworks gibt es seit einigen Jahren mit verschiedenen Zielsetzungen [3]. Bild 1 zeigt das qualitätsorientierte Data Governance Framework, dessen Ziel die Bereitstellung von Stammdaten mit hoher Qualität für alle Nutzenden ist. 
DasFramework ist in drei Ebenen gegliedert, in denen die sechs verschiedenen Handlungsfelder von Data Governance verortet sind. Als Querschnittsaufgabe, und damit eines der Hauptziele eines nachhaltigen Datenmanagements, ist Datenqualität genannt. Dieser Aspekt betrifft alle Ebenen und Handlungsfelder.

Die strategische Ebene adressiert die Datenstrategie. Die Verankerung in den Unternehmenszielen ist wichtig, um zu zeigen, dass Daten Teil des Wirtschaftsguts des Unternehmens sind (,Data as an asset ${ }^{\prime \prime}$ ) und damit einen wesentlichen Beitrag zur Erfüllung der (Umsatz-) Ziele leisten. Mit dieser Verankerung geht einher, dass die Implementierung eines MDM-Systems mit den notwendigen Ressourcen ausgestattet wird und das Top Level Management als Sponsor des Vorhabens auftritt.

Die Ebene der Organisation umfasst das Handlungsfeld Controlling. Das ist als Bindeglied zwischen der strategischen und organisatorischen Ebene zu verstehen. Das Controlling ermöglicht der Organisation, das Management der Stammdaten so zu steuern, dass die Anforderungen aller Nutzenden bedient werden. Kennzahlen messen den Grad der Zielerreichung und geben so Hinweise auf mögliche Optimierungen. Das schließt Kennzahlen zur Messung der Datenqualität mit ein. Ein weiteres Handlungsfeld auf dieser Ebene ist die Organisation des Datenmanagements, also die Frage nach der Definition von Rollen und Verantwortlichkeiten. Zudem werden auf dieser Ebene die Datenprozesse und Methoden definiert. Speziell geht es um die Datenproduktions- und Datenpflegeprozesse, die den Data Lifecyle vom Punkt der Entstehung oder Erhebung der Daten über deren Verarbeitung bis hin zur Archivierung und Löschung beschreiben.

Die Ebene der Informationssysteme spricht die Handlungsfelder Datenarchitektur und Systemarchitektur an. Bei der Datenarchitektur geht es um Datenmodelle und die logische Verteilung der Daten im Unternehmen. Die Handlungsebene der Systemarchitektur beschreibt, in welchen Anwendungs- systemen und Datenbanken welche Datenobjekte gespeichert sind und über welche Schnittstellen die Systeme miteinander verknüpft sind. Letztendlich ist eine gut aufgestellte System- und Datenarchitektur die technische Voraussetzung für ein fachlich effizientes Datenmanagement.

Als Querschnittsfunktion neben allen Ebenen steht die Datenqualität. Denn letztlich geht es beim Stammdatenmanagement in Kombination mit einer Effizienzsteigerung um eine hohe Qualität der Stammdaten. Ist diese nicht gewährleistet und wird diese nicht in den Handlungsfeldern adressiert, werden die Daten zwar gemanagt, aber ob die Qualität der Daten dann den Anforderungen der Nutzer genügt, darf infrage gestellt werden.

\section{Tipps zur MDM- Implementierung}

\section{Tipp 1: Festlegen einer Daten- strategie}

(Stamm-)Datenmanagement braucht einen Plan. Zu überlegen ist, welchen Stellenwert die Stammdaten im Wertegefüge des Unternehmens haben (sollen). Sind sie Teil des Wirtschaftsguts? Für welche Zwecke sollen sie in Zukunft eingesetzt werden? Ist eine Monetarisierung angedacht? Sollen die Daten mit externen Informationen angereichert werden? Gibt es regulatorische Vorgaben, die eingehalten werden müssen? All das sind Fragen, die im Rahmen einer Strategie beantwortet werden sollen und welche die Weichen für alle Folgeaktivitäten stellen. Nur wenn eine Strategie da ist, die von der Unternehmensleitung verabschiedet wurde und die im Unternehmen bekannt ist, kann diese umgesetzt werden. Der Strategie sind auch Hinweise zu entnehmen, wie sich das Unternehmen in den anderen Data-Go-
vernance-Handlungsfeldern aufstellen muss.

\section{Tipp 2: Aufbau einer Daten- management-Organisation}

In einer Datenmanagement-Organisation ist zu klären, wer für Daten zuständig ist und wer Entscheidungen über Daten im Unternehmen oder in den Fachbereichen trifft. Die Empfehlung: Aufbau einer DatamanagementOrganisation, welche die verschiedenen Hierarchie- und Entscheidungsebenen des Unternehmens abbildet und Festlegung, wer sich um welche Daten in welcher Verantwortung kümmert. Klassische Rollen dabei sind Chief Data Officer, Data Steward und Data Custodian (vgl. [3]). Ist die DatenmanagementOrganisation bekannt und die Rollen besetzt, können die Anforderungen an die Daten viel schneller geklärt, entschieden und umgesetzt werden.

\section{Tipp 3: Definition und Umsetzung von Datenproduktions-Prozessen}

Die Erfahrung zeigt, oft wird die Anlage von Stammdaten im gleichen System auf unterschiedliche Weise vollzogen. Soll ein neues MDM-System implementiert werden, müssen auch die Datenproduktions-Prozesse angepasst 
werden. Da die Prozesse die Qualität der Daten bestimmen, können Quality Gates zur Sicherung der Datenqualität bei der Anlage und Änderung der Stammdaten festgelegt werden. Bei der Definition und Umsetzung von Stammdatenprozessen ist die Empfehlung, die Prozesse mit den am Prozess beteiligten Mitarbeitenden neu zu definieren oder anzupassen. Auch die grafische Abbildung zur Nachvollziehbarkeit und Weitergabe an (neue) Kollegen ist wichtig. Eine einfache und verständliche Darstellung der Prozesse ist BPMN 2.0. Zusätzlich können die Prozesse mit weiteren Informationen angereichert werden. Das kann eine RACl-Matrix sein, aus der die Verantwortlichkeiten und Kommunikationswege eines Prozesses hervorgehen [4]. Sind alle Dokumente an zentraler Stelle abgelegt und verfügbar, können sich alle Mitarbeitenden, die mit den jeweiligen Prozessen zu tun haben, sehr schnell informieren und sind handlungsfähig.

\section{Tipp 4: Entscheidung für ein MDM- System}

Vor der Entscheidung für ein Stammdatensystem und dessen Einführung sollte die Zielsetzung des Managements von Stammdaten aus der Datenstrategie abgeleitet werden. Als Stammdatensysteme kommen neben den MDM-Systemen auch CRM-Systeme für Geschäftspartnerdaten und ERP-Systeme für Materialstammdaten in Frage. Im Gegensatz zu MDM-Systemen sind CRM- bzw. ERP-Systeme stark auf die entsprechenden Prozesse aus den Bereichen Geschäftspartner und Materialien zurechtgeschnitten. Es gibt vorgegebene Datenmodelle und Workflows zu deren Erstellung und Bearbeitung. Bei MDM-Systemen müssen die Datenmodelle zunächst modelliert werden. Auch werden in MDM-Systemen keine typischen Geschäftsprozesse abgebildet. Stammdatenmanagement-Systeme werden dann ihrem Namen gerecht, wenn sie tatsächlich als zentrales System für sämtliche Stammdaten eingesetzt werden. Die Empfehlung lautet: ein System finden, was die Ziele der Da- tenstrategie in den kommenden Jahren am besten abbildet.

\section{Tipp 5: Datenqualität als tragende Komponente}

Wer von Stammdaten spricht, spricht von Datenqualität. Da die Stammdaten an zentraler Stelle gemanagt werden und von dort aus an andere Datensenken verteilt werden, ist es unabdingbar, die Anforderungen an die Qualität der Daten von allen Bereichen abzubilden, die die Stammdaten nutzen. Ist das nicht der Fall, entwickeln sich sehr schnell individuelle Datensilos wie Excel-Tabellen, die auf die Bedürfnisse der jeweiligen Arbeiten angepasst sind. Ist nicht bekannt, wie gut oder wie schlecht die Qualität der Daten ist, also wie geeignet sie für den Verwendungszweck sind, geht das Vertrauen in die Stammdaten verloren. Somit ist eine gute und definierte Datenqualität bei Stammdaten unabdingbar (z.B. [5]). Und diese kann in der Datenstrategie verankert sein, in der Organisation in der Verantwortung von definierten Rollen liegen, in den Prozessen in Form von Quality Gates definiert werden und von MDM-Systemen systemseitig umgesetzt werden. Die Qualität der Stammdaten sollte in jedem Handlungsfeld berücksichtigt werden.

\section{Fazit}

Der Erfolg einer MDM-Implementierung misst sich an der langfristigen und nachhaltigen Nutzung des Systems und der darin gehaltenen Daten. Datenqualität ist dafür ein entscheidender Erfolgsfaktor. Die oben aufgeführten Tipps geben konkrete Hinweise, was bei einer Implementierung unbedingt beachtet werden sollte. Adressiert man all diese Hinweise, bekommen die Stammdaten den passenden Rahmen, um ihren Teil zum Erfolg des Unternehmens beizutragen.

\section{Literatur}

[1] R. Scheuch, T. Gansor, und C. Ziller, Master Data Management: Strategie, Organisation, Architektur, 1. Aufl. Heidelberg: dpunkt. Verl, 2012.
[2] C. Klingenberg und K. Weber, "Informations- und Datenmanagement", in Handbuch IT-Management - Konzepte, Methoden, Lösungen und Arbeitshilfen für die Praxis, 7. Auflage., München: Hanser, 2020, S. 225-280.

[3] K. Weber und C. Klingenberg, Data Governance: Der Leitfaden für die Praxis. Hanser, 2020.

[4] C. Klingenberg und T. Brandstätter, ,Einführung eines Datenqualitätsmanagements durch Etablierung von Quality Gates in der Datenproduktion", BI-SPEKTRUM, Bd. 5/2020, S. 19-22, Dez. 2020.

[5] K. Hildebrand, "Management der Materialstammdaten in SAP ${ }^{\oplus}$-Systemen", in Datenund Informationsqualität, K. Hildebrand, M. Gebauer, und M. Mielke, Hrsg. Wiesbaden: Springer Fachmedien Wiesbaden, 2021, S. 307-319.

\section{Schlüsselwörter:}

Data Governance, Master Data Management, qualitätsorientiertes Data Governance Framework, Datenqualität

\section{Data Governance as a Success}

Factor for MDM Implementations

With Master Data Management (MDM) companies want to increase efficiency and transparency when managing their master data, while, at the same time, minimizing risks. Master data, such as customer or product data, will become an important "asset" within the company. The article reveals practical hints that show how MDM implementations become sustainable, so that master data contribute to the success of the company. The article presents the qualityoriented Data Governance Framework. When using the framework as a guidance, all aspects of an MDM implementation will be covered, including strategy and organization. Due to the orientation towards data quality all areas within a company may use master data to their benefit.

Data Governance, Master Data Management, quality-oriented Data Governance Framework, Data Quality

Kontakt:

Dr. Christiana Klingenberg msg systems ag Max-Planck-Straße 40 50354 Hürth/Köln E-Mail: christiana.klingenberg@ msg.group 